\title{
Duplication of the rectum
}

\author{
M. J. KeLLY \\ M.Chir., F.R.C.S., M.R.C.P.(U.K.) \\ W. G. EVERETT \\ M.Ch., F.R.C.S.
}

Addenbrooke's Hospital, Hills Road, Cambridge

\begin{abstract}
Summary
A case of duplication of the rectum is presented and the literature reviewed.
\end{abstract}

\section{Case report}

A 35-year-old industrial chemist was admitted for treatment of increasingly frequent episodes of perianal pain and discharge. The pain occurred every few months, building up in intensity until a peri-anal discharge of faeculant material brought relief. $\mathrm{He}$ had suffered similar episodes all his life.

At the age of 4 years he was admitted to The Hospital for Sick Children, Great Ormond Street, for the investigation of a peri-anal lesion causing pain and discharge. On examination under anaesthesia, a small orifice lying $0.5 \mathrm{~cm}$ behind and slightly to the right of the anus was discovered. A probe could not be inserted and it was presumed that he had a healing anal fistula.

During childhood he experienced infrequent bouts of peri-anal pain relieved by the discharge of faeculant material. Between episodes he had no anal symptoms. Over the previous two years these bouts had become more frequent and of longer duration. Latterly they were occurring once a month and the discharge of faeculant material, which was never blood-stained, ceased to bring complete relief. He habitually felt pain before defaecation, maximal during the act, and receding during the next few hours. The bowel actions were otherwise quite normal. There had been a recent decrease in sexual drive, but there were no urological symptoms and his general health was good.

He was admitted in July 1974 and examination revealed a fit-looking man whose only abnormalities were confined to the perineum. There was a sinus opening $1 \mathrm{~cm}$ behind and to the right of the anus. On rectal examination a firm non-tender fusiform mass $10 \times 5 \times 5 \mathrm{~cm}$ could be felt bulging forwards into the rectum. It appeared to be attached posteriorly to the sacrum but the mucosa of the rectum overlying the mass felt mobile and normal. On firm pressure, the mass could be impressed by the examining finger giving the sensation that faecal matter was being palpated. Rectal pressure did not produce any discharge from the sinus. The external genitalia appeared normal.
Standard biochemical investigations were normal. Sigmoidoscopy to $15 \mathrm{~cm}$ showed normal mucosa in a displaced rectum. X-ray of the pelvis (Fig. 1) revealed a defect in the right wing of the sacrum and an abnormal neural arch in the 5th lumbar vertebra. Barium studies (Fig. 2) demonstrated a fusiform post-rectal mass displacing the rectum forwards. A sinogram, performed by inserting a cannula into the peri-anal orifice (Fig. 3), showed that the periphery of the mass was partially outlined and that it contained solid material. A myelogram (Fig. 4) failed to demonstrate any connection between the sacral meninges and the mass.

The size, shape and position of this mass were confirmed by examination under anaesthesia. A transrectal needle biopsy of the mass was performed. This showed the presence of smooth muscle but failed to provide any further diagnostic information.

In December 1974 the pre-sacral mass was explored with the patient in the lithotomy position, using a peri-anal approach. A curved postanal incision displayed a fusiform mass situated between the sacrum behind and the rectum in front. The rectal wall was dissected off the mass with relative ease and no intercommunication found. At this point in the operation the wall of the mass was ruptured and a large quantity of what appeared to be inspissated faecal matter was evacuated. The lining of the mass, which extended upwards and to the right, was dissected off the surrounding tissues. At its upper extent it entered a circular bony defect in the right side of the sacrum approximately $15 \mathrm{~cm}$ from the anal margin. No serious haemorrhage was encountered. The wound was loosely approximated around a corrugated rubber drain.

Recovery was complicated by a transient hypotensive and pyrexial episode. Although blood culture was sterile, this episode was presumed to be due to a septicaemia and it responded to transfusion and a course of co-trimoxazole. The patient was discharged after two weeks, and the wound had fully healed two months after the operation.

Histology of the resected specimen showed debris, smooth muscle surrounding a flattened membrane suggestive, but not diagnostic, of an atrophic epithelium. 


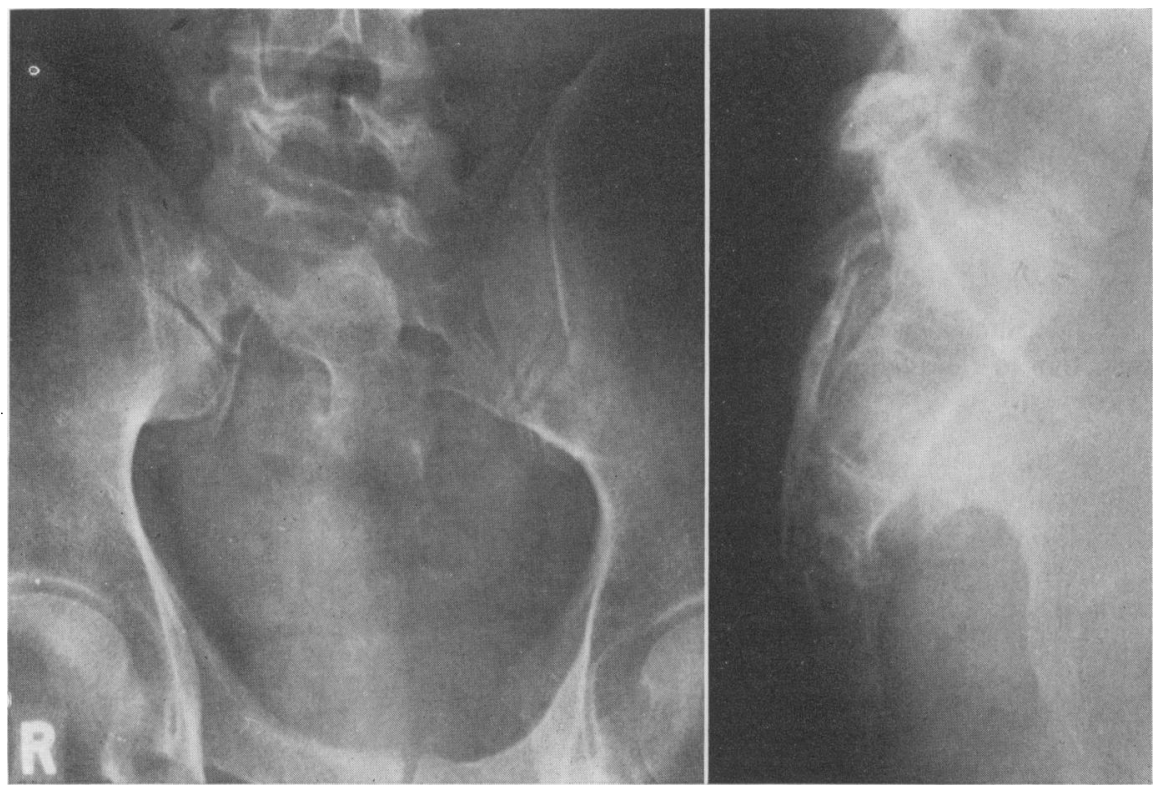

FIG. 1. Plain X-ray of the pelvis showing sacral agenesis.

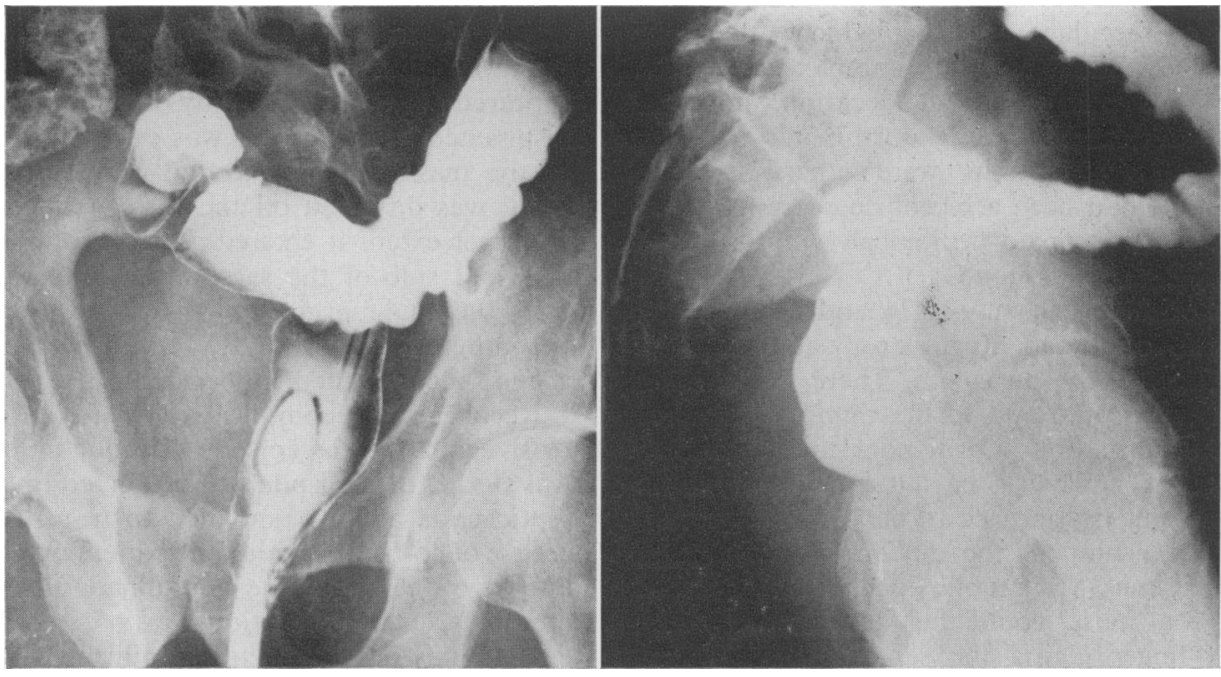

Fig. 2. Barium enema showing lateral and anterior displacement of the rectum. 


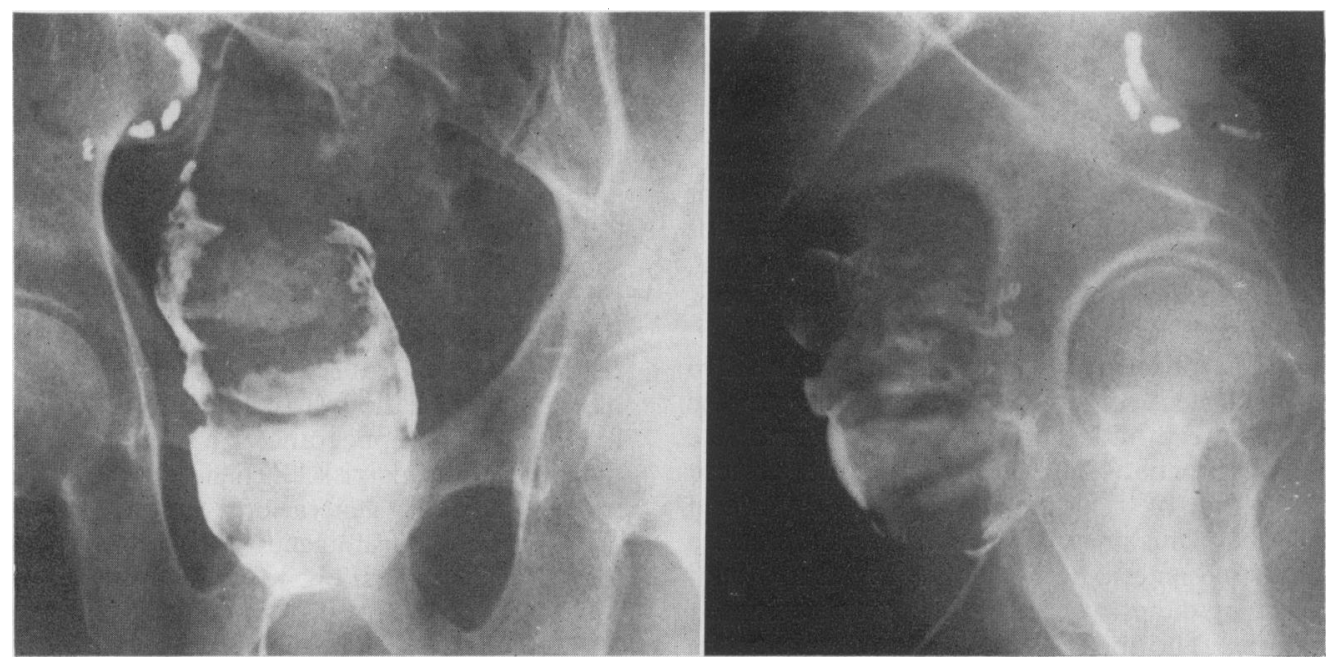

FIG. 3. Sinogram, antero-posterior and lateral views.

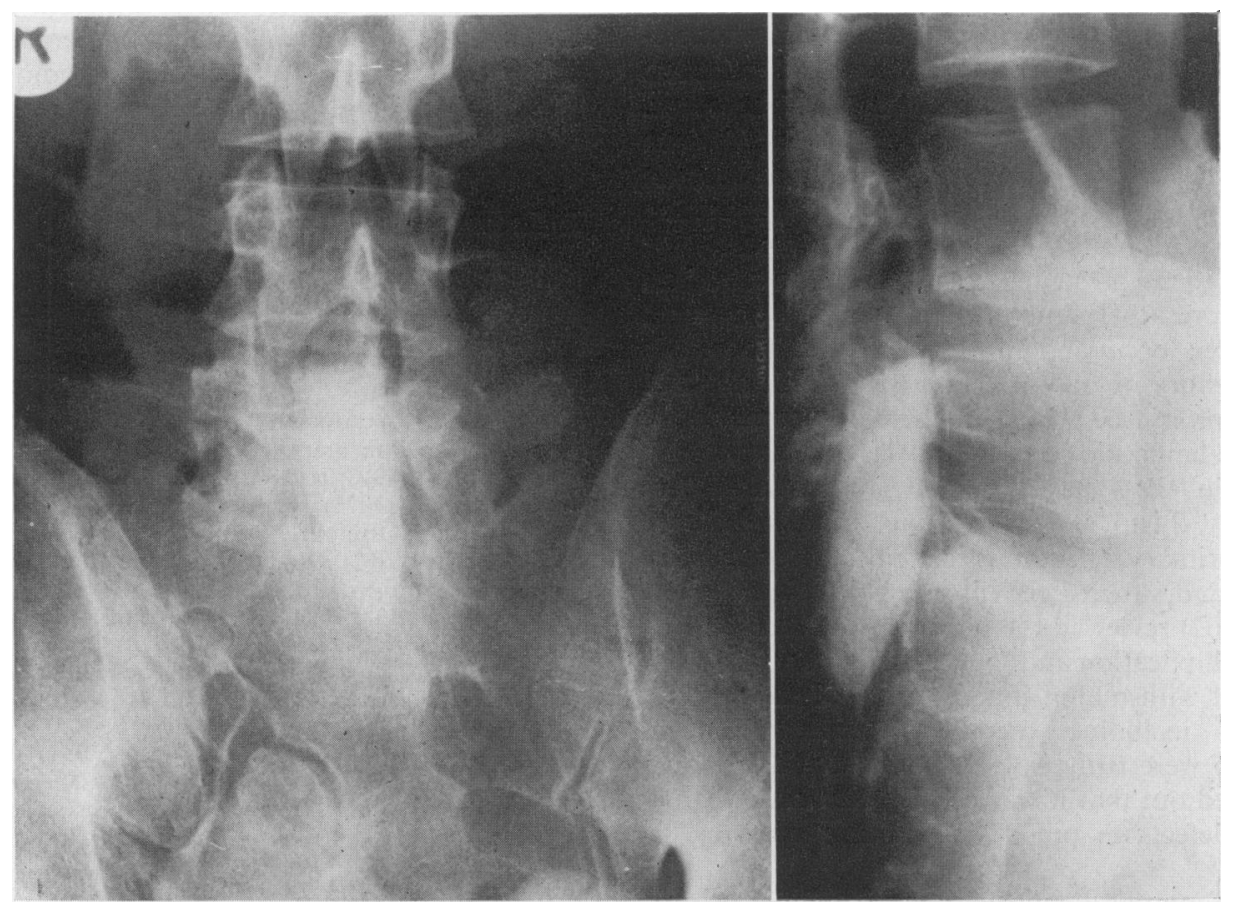

FIG. 4. Myelogram showing no connection between the rectal duplication and the meninges. 
Three years later the patient remains well and has had no recurrence. His continence is unimpaired and his sexual function has returned to normal.

\section{Discussion}

Duplication of the rectum is thought to be a rare embryological abnormality. The literature contains a number of case reports in which this anomaly is described in both adults and infants (Mousseau, Konno ard Hanson, 1963; Kraft, 1962; Gross, Holcombe and Farber, 1952).

One of the problems of these abnormalities is to explain their lack of constant relationship to the kowel mesentery and the presence of a complete intact muscle coat. The earliest of the current theories is that of 'budding diverticula' (Lewis and Thyng, 1908). This postulates that small bulges arise on the antemesenteric border of the bowel and budoff, becoming either true diverticula or occasionally duplications. The most accepted current theory is that of Bremmer (1944) who divided duplications into two types, spherical and tubular. The spherical he considered understandable in terms of Lewis' concept of budding; the tubular ones, however, he explained by a new vacuolation theory. It was known that during development many hollow viscera, including the gut, passed through a proliferative phase when their lumen was occluded. These cores of cells then vacuolated and the vacuoles coalesced to reestablish a lumen. He suggested that if this vacuolation should become disordered, tubular duplication incorporating complete muscle coats might occur. Other theories such as 'caudal twinning' (von Zwalenburg, 1952), persistent omphalo-mesenteric remnant (Fitz, 1884) and notochordal separation (Veeneklaas, 1952) have fallen into disfavour.

Kraft (1962) presented a case of removal of an inflamed presacral structure, believed to be a rectal duplication, occurring in a 34-year-old housewife after a history of only 2 years. Before this she had been symptom-free, which might suggest some doubt about the embryological origin. The present case is unusual in that the childhood investigation and subsequent history of symptoms clearly indicate that the primary defect must have been present since infancy and, therefore, presumably since birth. Kraft (1962) reviewed the literature of seventy cases of rectal duplication and found that thirty-eight were associated with major abnormalities of the hindgut structure, including the genito-urinary system; thirty-two were truly isolated rectal malformations. He pointed out that it was not uncommon for these isolated defects to present, apparently de novo, in adult life.

The differential diagnosis of a retro-rectal or presacral mass includes post-rectal abscess, teratoma, dermoid cyst, chordoma, sacral meningocele, as well as soft tissue tumours such as lipoma, lipomosarcoma and neurilemmoma (Leading Article, 1971). The commonest retrorectal tumours are teratoma, dermoid cyst and chordoma.

Sacral agenesis, first described by Hohl in 1852, has long been known to be associated with hindgut and ano-rectal malformations. Williams and Nixon (1957) reported eight cases of imperforate anus with sacral agenesis and reviewed forty-one cases from the literature. They concluded that loss of more than one complete sacral segment was associated with a high risk of bladder disturbance, whilst failure of fusion of segment 1 to the rest of the sacrum, which might have a similar appearance on superficial scrutiny, did not carry this risk. Kenefick (1973) reported a family of four generations among whom nine members had sacral agenesis and four of these were found, on investigation, to have presacral tumours. Two tumours were excised (one meningocele and one benign dermoid cyst) and two were left in situ to be followed as out-patients. He suggested that sacral agenesis may be transmitted by a sex-linked dominant gene. None of the four pre-sacral masses has been shown to be a rectal duplication. As far as the authors have been able to discover, the association of sacral agenesis with rectal duplication has not been previously reported.

Most authorities are agreed that the treatment of choice in rectal duplication is excision. A perineal approach is recommended and, as demonstrated in the present case, it is possible to remove a mass extending as high as the second sacral segment without difficulty.

\section{References}

BREMMER, J.L. (1944) Diverticula and duplications of the intestinal tract. Archives of Pathology, 38, 132.

Fitz, R.H. (1884) Persistent omphalomesenteric remains; their importance in the causation of intestinal duplication, cyst formation and obstruction. American Journal of Medicine, 88, 30 .

Gross, R.E., Holcombe, G.W. \& Farber, S. (1952) Duplications of the alimentary tract. Pediatrics, 9, 449.

HoHL, A.F. (1852) Zur Pathologie den Beckers, pp. 61.

KENEFICK, J.S. (1973) Hereditary sacral agenesis associated with presacral tumours. British Journal of Surgery, 60, 271.

KRAFT, R.O. (1962) Duplication anomalies of the rectum. Annals of Surgery, 155, 230.

LEADING ARTICLE (1971) Retrorectal tumours. British Medical Journal, 3, 261.

LEWIS, F.T. \& THYNG, F.W. (1908) The regular occurrence of intestinal diverticula in the embryos of pig, rabbit and man. American Journal of Anatomy, 7, 505.

Mousseau, L.P., Konno, T. \& Hanson, S. (1963) Duplication of the rectum. Canadian Journal of Surgery, 6, 438.

VeeneklaAs, G.M.H. (1952) Pathogenesis of intrathoracic gastrogenic cysts. American Journal of Diseases of Children, 83, 500 .

von ZWalenburg, B.R. (1952) Double colon. American Journal of Roentgenology, 68, 22.

Williams, D.I. \& Nixon, H.H. (1957) Agenesis of the sacrum. Surgery, Gynecology and Obstetrics, 105, 84. 